\section{AB0829 OSTEOGENESIS IMPERFECTA : EVOLUTION OF THE BONE MINERAL DENSITY AND EVALUATION OF FRACTURE RISK UNDER BISPHOSPHONATES}

Mohamed Amine El Achek, Mahbouba Jguirim, Amel Farhat, Saoussen Zrour Ismail Bejia, Mongi Touzi, Naceur Bergaoui. Fattouma Bourguiba University Hospital, Rheumatology, Monastir, Tunisia

Background: Osteogenesis imperfecta (OI), or Lobstein's disease, also known as 'Glass bone disease' is a rare genetic disorder predisposing to low bone mass with impaired bone microarchitecture and abnormal quality of bone material, causing fracture susceptibility and bone deformities. Objectives: The main objective of our work was to study the clinical and para-clinical characteristics, and global management, of adult patients and children with $\mathrm{OI}$ in a rheumatology departement by comparing them with data from the litterature.

The secondary objectives were to study the evolution of the bone mineral density and to evaluate the fracture incidence under treatement by Bisphosphonates.

Methods: This is a cross-sectional, observational, descriptive, analytical and monocentric study of subject with Ol followed at Fattouma Bourguiba University Hospital in Monastir, Tunisia

Results: We collected 13 patients with Ol, including 5 children and 8 adults. Seven patients were female and 6 were male, including 12 patients with type IB and one with type IIIB. Three adult patients had a family history of Ol. For children, it was sporadic and the genetic survey did not reveal similar cases in the family. The mean age of clinical onset of the disease was 9 years and 3 months [0-33 years] with a mea nage at diagnosis of 15 years and 7 months [14 months- 40 years]. The clinical manifestations were dominated by osteo-articular symptoms. Chronic mechanical bone pain related to fractures was present in eight patients. Twelve patients had a history of fracture. These were repeated fractures without trauma or minimal traumain the majority of cases mainly in the lower limbs. The first fracture site was the femur associated with other fracture sites (there were no fractures in the pelvis, hands and skull). Deformities were observed in 7 patients, mainly in the limbs (6 patients), in the spine (2 patients) and in the thorax (1 patient)

In our study the frequency of osteoporosis and/or osteopenia regardless of the mesured site and using the T-score value for women $\leq-2,5$ and the Z-score $\leq-2$ for children and men was $84,62 \%$. All patients received Aredia treatment according to Glorieux's protocol with calcium and vitamin D supplementation. The treatment was also multidisciplinary. Bisphosphonate treatment was well tolerated for the majority of patients. There was a decrease in the number of fractures and also a densitometric gain objectivised for the majority of patients

Conclusion: Our results confirm the efficacy of BP in patients with $\mathrm{Ol}$ regardless of age group. Short-term adverse are rare and transient.

\section{REFERENCE}

[1] R. Morello, Osteogenesis imperfecta and therapeutics, Matrix Biol. (2018). doi:10.1016/..matbio.2018.03.010.

Disclosure of Interests: None declared

DOI: 10.1136/annrheumdis-2019-eular.5041

\section{AB0830 CG AND DMARDS INDUCED BMD CHANGES IN PRE-, POSTMENOPAUSAL WOMEN AND MAN WITH RHEUMATOID ARTHRITIS (RA)}

Olena Garmish, Andrii Romanovskiy. National Scientific Center "M.D. Strazhesko Institute of Cardiology", Kiev, Ukraine

Background: Risk factors for GC-induced fracture include low bone strength at the beginning of GC treatment and the rate of decline in bone mass during treatment, which is largely determined by the dose and duration of GC use [1]. The absolute risk of future fracture in an individual is substantially influenced by demographic and other characteristics (age, race, sex, and concomitant OP risk factors). For these reasons, it is important to identify differences in BMD changing depends on sex and reproductive status.

Objectives: To compare the BMD changes in man, pre- and postmenopausal women with RA depends on different treatment regiments.

Methods: The study was performed on 145 patients: 117 women (mean age $45.4 \pm 13.0$ years, mean disease duration $9.7 \pm 7.7$ years, $41 \% \quad(n=48)$ postmenopausal) and 28 man (mean age $45.5 \pm 17.5$ years, mean disease duration $5.7 \pm 4.8$ years) with RA. Female patients were divided in two groups by menopause: premenopausal (PreM) in mean age $36.9 \pm 9.3$ years and postmenopausal (PM) in the mean age $57.6 \pm 5.9$ years. $68.4 \%$ women and $64.3 \%$ man received prednisolon $\leq 10 \mathrm{mg} /$ day during 5.67 \pm 4.82 and $3.5 \pm 5.9$ years respectively. $60.7 \%$ of men received MTX, $50 \%$ in combination with CGs. Among the women group $87 \%$ PreM patients received MTX, 58.3\% in combination with GCs and $46.4 \%$ with biologics. $85.4 \%$ PM women took MTX, $68.8 \%$ in combination with GCs and $33.3 \%$ - with biologics. BMD was measured in 3 part of the skeleton: hip (total and neck), lumbar spine, distal part of forearm.

Results: BMD was decreased in $44.5 \%$ of women and $42.9 \%$ of man BMD of hip, lumbar spine, distal part of forearm were respectively decreased in $26.1 \%, 26.1 \%, 18.8 \%$ PreM women and $66.7 \%, 70.8 \%$, $79.2 \%$ PM women. $39.3 \%$ of man had decrease BMD in the hip and $42.8 \%$ - in the lumbar spine. According to logistic regression in all women group the fact of $C G$ intake was strong associated with $B M D$ decrease in the hip (total and neck) and lumbar spine $(r=-0.25, p<0.05)$, in man no association with the fact of $C G$ intake was found. The effect of CGs on BMD in man was cumulative dose dependent, negative correlation between treatment duration and low hip neck $(r=-0.44, p<0.05)$ and lumbar spine BMD $(r=-0.5, p<0.05)$ was detected. In PreM women only association between CG therapy and decreased total and neck hip BMD $(r=-0.33, p<0.05)$ was detected, any relationship with cumulative dose and treatment duration wasn't found. Only in PM patients BMD decreasing depends on average cumulative dose and treatment duration. MTX intake was negatively correlated with BMD only in PM women in total hip and lumbar spine $(r=-0.37, p<0.05)$ and in men in the distal part of forearm $(\mathrm{Z}=-2.46, \mathrm{p}<0.05)$. In $\mathrm{PM}$ women $\mathrm{BMD}$ in total hip and distal part of forearm was significantly higher in $\mathrm{MTX}+\mathrm{CG}+$ biologics group than without biologics $(r=0.38, p<0.05)$. The level of PTG was significantly higher in PM patients on GCs and was negatively correlation with cumulative dose $(r=$ $0.77, \mathrm{p}<0.05)$. In PreM women GCs intake was negatively correlated with C-TP level $(r=-0.37, p<0.05)$ and positively with RANKL level $(r=0.35$, $\mathrm{p}<0.05$ ).

Conclusion: A sexual differences in BMD changes during different treatment regiments was observed in different parts of the skeleton. In man BMD lost in hip and lumbar spine depends on GCs treatment duration and on in the distal part of forearm on MTX intake. In PM women BMD decreasing was strong associated with GC and MTX cumulative dose and treatment duration.

\section{REFERENCE}

[1] Lenore Buckley, Gordon Guyatt, Howard A. Fink, et al. 2017 American College of Rheumatology Guideline for the Prevention and Treatment of Glucocorticoid-Induced Osteoporosis Arthritis Rheum Vol. 69, No. 8 , August 2017, pp 1521-1537.

Disclosure of Interests: None declared DOI: 10.1136/annrheumdis-2019-eular.4327

\section{AB0831 PERSPECTIVE ON THE CLINICAL PRACTICE MANAGEMENT OF HYPOVITAMINOSIS D FROM A MEETING OF ITALIAN EXPERTS}

Alessandro Giollo ${ }^{1}$, Ombretta Viapiana ${ }^{1}$, Angelo Fassio ${ }^{1}$, Francesco Bertoldo ${ }^{2}$ Vania Teresa Braga ${ }^{3}$, Maria Luisa Brandi ${ }^{4}$, Stefano Calvieri ${ }^{5}$, Luisella Cianferotti ${ }^{6}$, Annamaria Colao ${ }^{7}$, Patrizia D'amelio ${ }^{8}$, Giovanni Mario D'avola ${ }^{9}$, Luca Degli Esposti ${ }^{10}$, Bruno Frediani ${ }^{11}$, Sandro Giannini ${ }^{12}$, Andrea Giusti ${ }^{13}$, Andrea Giustina ${ }^{14}$, Stefano Gonnelli ${ }^{11}$, Nazzarena Malavolta ${ }^{15}$, Claudio Marcocci ${ }^{16}$, Salvatore Minisola ${ }^{5}$, Nicola Napoli ${ }^{17}$, Ranuccio Nuti ${ }^{1{ }^{1}}$, Giovanni Passeri ${ }^{18}$, Maurizio Rossini ${ }^{1}$, Luigi Sinigaglia ${ }^{19}$, Francesco Vierucci $^{20}$, Davide Gatti ${ }^{1}$, D.battito Group. ' University of Verona, Rheumatology Unit, Department of Medicine, Verona, Italy; ${ }^{2}$ University of Verona, General Medicine and Atherosclerosis Unit, Verona, Italy, ${ }^{3}$ ULSS9 Scaligera Verona, Centro per la diagnosi e la terapia per l'Osteoporosi, Verona, Italy; ${ }^{4}$ University of Florence, Departmento of Internal Medicine, Florence, Italy, ${ }^{5}$ Università "Sapienza" di Roma, Roma, Italy; ${ }^{6}$ University of Florence, Florence, Italy; ${ }^{7}$ Università di Napoli Federico II, Napoli, Italy; ${ }^{8}$ Università di Torino, Torino, Italy, ${ }^{9}$ ASP 3 Catania, Catania, Italy;

${ }^{10} \mathrm{CliCon}$ srl, Health, Economics and Outcomes Research, Ravenna, Italy,

${ }^{11}$ University of Siena, Siena, Italy, ${ }^{12}$ University of Padova, Padova, Italy;

${ }^{13}$ Ospedale Galliera, Genova, Italy, ${ }^{14}$ Università Vita e Salute San Raffaele, Milano, Italy; ${ }^{15}$ University of Bologna, Bologna, Italy, ${ }^{16}$ University of Pisa, Pisa, Italy;

${ }^{17}$ Università Campus Bio-Medico di Roma, Roma, Italy, ${ }^{18}$ University of Parma, Parma, Italy; ${ }^{19}$ Centro Specialistico Ortopedico Traumatologico "Gaetano Pini", Milano, Italy; ${ }^{20}$ ASL 2 Lucca, Lucca, Italy 